\title{
Breaking Well-Formed Opinions and Mindsets by Designing with People Living with Dementia
}

\author{
Paul A. Rodgers and Euan Winton
}

\begin{abstract}
This paper presents ongoing research that highlights how design thinking and acting can contribute significantly to breaking down preconceived ideas about what people living with dementia are capable of doing. The research, undertaken in collaboration with Alzheimer Scotland and other dementia organisations across the UK, has adopted a range of disruptive design interventions to break the cycle of wellformed opinions, strategies, mindsets, and ways-of-doing, that tend to remain unchallenged in the health and social care of people living with dementia. The research has resulted in a number of co-designed interventions that help change the perception of dementia by showing that people living with dementia can offer much to UK society after diagnosis. Moreover, it is envisaged that the co-designed activities and interventions presented here will help reconnect people recently diagnosed with dementia to help build their selfesteem, identity and dignity and help keep the person with dementia connected to their community, thus delaying the need for formal support and avoid the need for crisis responses. The paper reports on three design interventions where the authors have worked collaboratively with nearly 200 people diagnosed with dementia across the UK in co-design and development activities. The paper concludes with a number of innovative recommendations for researchers when co-designing with people living with dementia.
\end{abstract}

\section{Background}

In the UK life expectancy over the last few decades has risen steadily. Today, females born in 2015 can expect to live 82.8 years from birth (4 years more than females born in 1991) and males have seen a greater increase in life expectancy of 5.7 years, from 73.4 years for males born in 1991 to 79.1 years for males born in 2015. Life expectancy in the UK is projected to continue increasing; with life expectancy at birth for females projected to be 85.1 years by 2026 and 86.6 years by 2036. Males are also projected to live longer, increasing to 82.1 years by 2026 and 83.7 years by 2036. Improved healthcare and lifestyles, especially for those aged 65

Paul A. Rodgers and Euan Winton, Imagination, Lancaster University, p.rodgers@1ancaster.ac.uk \& e.winton@1ancaster.ac.uk 
and over, is the main reason for the increase in life expectancy (Office for National Statistics, 2017).

The consequences of these population changes in the UK, however, means the shape of the UK population is transforming with those of a working age shrinking whilst those of a pensionable age is increasing. While a larger population can increase the size and productive capacity of the workforce, it also increases significant pressures and questions the sustainability to provide social services such as education, health and social care, and housing. The rise in life expectancy in the UK is a key factor in the forecasted rise of people living with dementia in the UK. The number of people with dementia in the UK is forecast to increase to over 1 million by 2025 and over 2 million by 2051 . By the year 2030 , over $80 \%$ more people aged 65 and over will have some form of dementia compared to 2010 .

In the UK, dementia and how we respond to it has reached a crisis point. It is a problem that improved public awareness or a better diagnosis alone will not solve. The management of long-term conditions associated with dementia is the key challenge facing the health and social care system in the UK. The UK Government believes we need to see profound changes to the way we view the person living with dementia as well as the overall system of health and social care (All Party Parliamentary Group on Dementia 2016). With this in mind, the three co-design projects presented in this paper show that people living with dementia can continue to make a significant contribution to society after diagnosis. The approach taken here actively involved a range of stakeholders in the design process such as care workers, people living with dementia, and their family and friends to help ensure the three codesign projects met their needs and would be valuable and useful. The three codesign projects were carefully developed to be more appropriate to people living with dementia and their emotional and practical needs. Moreover, the three codesign projects aim to help reconnect people recently diagnosed with dementia to build their self-esteem, identity and dignity and keep the person with dementia connected to their community.

\section{The Nature of Dementia}

Dementia is a broad umbrella term used to describe a range of progressive neurological disorders. There are many different types of dementia and each person will experience their dementia in their own unique way. Common symptoms of dementia can include problems with short term memory where new information is difficult to retain. People with dementia can get lost in seemingly familiar places, may experience confusion with names, and may also experience confusion in environments which are unfamiliar to them. As a result, people with dementia may lose interest in engaging with others socially, so a person with dementia may become quieter and more introverted, and their self-confidence might become affected. Amongst older people, dementia makes the largest contribution to the need for care, much more so than other types of impairment and chronic disease (Prince et al. 2013). This demand for health and social care services will continue to increase as a result of demographic changes. Responding to this challenge will require innovative 
ways of supporting people with dementia to live well from the early stages of the illness. Receiving a diagnosis of dementia creates a 'biographical disruption', with the chronically ill 'observing their former self-images crumbling away' (Bury 1982). People need support from the point of diagnosis to come to terms with this life altering event, remain connected to their community and enable them to live well with this long-term illness. However, people typically do not receive support until the illness is advanced and often at the point of crisis (Alzheimer Scotland 2008). This pattern is becoming more acute as a result of pressure on health and social care budgets.

Philosophical debates on dementia have largely focused around the fundamental nature of being and what constitutes personhood. The failure to recognise personhood and the negative impact of inappropriate care giving can result in 'malignant social psychology', which includes labelling, disempowerment, infantilisation, invalidation and objectification. One reason behind this malignance is failing to see a person and not showing the respect that properly accords a person (Kitwood 1990). Even when a person seems to have lost a significant part of what made them a unique individual, core elements of their identity will remain. These characteristic gestures and ways of doing things are what keep alive the sense of the individual they once were, even if the more sophisticated levels of that individual have been removed. This has important implications for the approach to providing support and what people require in addition to the basics of daily living. A person's sense of self and self-respect can be fostered through 'reinforcing any remaining elements of conscious self-identity'; less conscious elements in a person's identity can be preserved through physical surroundings to retain 'physical links with their past, which help to support a sense of personhood' (Matthews 2006). Whilst mood and behaviour may be profoundly affected, personhood is not; the individual remains the same equally valuable person throughout the course of the illness. Interventions to support the person with dementia should honour their personhood and right to be treated as a unique individual.

\section{Designing with People Living with Dementia}

The key aim of this ongoing research is to develop a number of disruptive design interventions (e.g. products, systems, services) that break the cycle of well-formed opinions, mindsets, and ways-of-doing, that tend to remain unchallenged in the health and social care of people living with dementia. Many misconceptions surround people living with dementia, which can result in the perpetuation of stigma, isolation, and generally negative reactions. The idea that nothing can be done to help people with dementia often leads to feelings of hopelessness and frustration (Batsch and Mittelman, 2012). Many people living with dementia have a sense of inadequacy and low self-esteem. They perceive their status within society has been reduced as a result of their diagnosis (Katsuno, 2005). In the main, people living with dementia are not considered capable of designing new products or services. This ongoing work sets out to directly challenge this assumption. The design interventions presented here (i.e. Disrupting Dementia tartan, Designed With Me, and 75BC) have all been 
devised and undertaken from a 'designing with' perspective where the user is not viewed as a 'subject' but rather as an active 'partner' in the project (Sanders and Stappers 2014). The approach taken here encourages the development of richer, more varied solutions to everyday issues by emphasising fun (Bisson and Luckner 1996), 'safe failure', and doing things in ways that those working with people with dementia would not normally do. The three co-design projects presented here adopt a largely interventionist approach, which are based on a number of emerging theories emanating from research in economics, business and design (Christensen and Overdorf 2000; Scharmer 2011; Rodgers and Tennant 2014) that celebrate jumping straight in, doing things in order to learn new things, and valuing failure.

Co-design has been widely used in the commercial sector. However, recent research shows that co-design is increasingly used in the public sector, including the the third sector, as a way of engaging citizens in design exploration (Lam et al. 2012). Many co-design techniques and tools, however, assume particular skills, expertise, and processes that rely on certain levels of communication, cognitive, and creative skills on the part of the participants. As such, many well-established co-design tools and techniques may not be appropriate and need adjustment (Wilson et al. 2015). Indeed when working with people with cognitive and other impairments such as dementia, researchers may have to develop and adopt highly individual co-design approaches and methods (Hendriks et al. 2015). The motivation behind the three projects presented here has been to ensure that everyone involved is engaged fully. As such, great care has been taken to consult with people living with dementia, their family members, and care support workers about how they wanted to be involved throughout the projects before they started. In particular, it was vital that the planned co-design projects supported the person living with dementia and that it paid respect to their personhood and their right to be treated as a unique individual (Kinnaird 2012). A co-design approach acknowledges that each individual has their own strengths and weaknesses that they bring to the co-design process. Consequently, the three projects presented here have been carried out with people living with dementia who, it is hoped, will benefit from the experiences. Indeed, the key objective behind this work is to care better for people living with dementia and break down widely held and largely negative preconceived ideas about what people living with dementia are capable of doing.

\subsection{Disrupting Dementia Tartan}

The first of the three projects presented here is the Disrupting Dementia tartan. This co-design project involved over 130 people living with dementia taking part in codesign workshops held all over Scotland. The project involved in excess of 1900 miles of travel, over 80 hours spent travelling, and using over half a kilometre of coloured ribbon in the creation of the participants' tartan design prototypes. The main aim of the Disrupting Dementia tartan design project is to help change the perception of dementia by showing that people with dementia can offer much to UK society after diagnosis. Specifically, that people living with dementia are capable of designing a new product that will be sold all over the world. Moreover, this project 
will help people recently diagnosed with dementia build their self-esteem, identity and dignity and help ensure that every person living with dementia and their families' quality of life and resilience is maximised. In a co-design project such as this one, it is important that the designer does not take an overly dominant role. The instigator of the co-design project should be transparent about the project's objectives and clearly articulate the reasons behind embarking on a co-design project. In other words, the project rationale should always be known from both sides. The goal is to achieve something like a symbiotic collaboration-a mutually beneficial relationship between those involved. Van Klaveren (2012) suggests such an ethical and transparent approach is the foundation for a truly symbiotic co-design relationship.

In this project, each tartan co-design workshop commenced with a short presentation of the basic rules associated with the creation of the Disrupting Dementia tartan. Working closely with Alzheimer Scotland staff and family members, care was taken to ensure that the language used during the workshop was supportive and not offensive to people living with dementia. Also, the researcher supported by care support workers ensured that everyone taking part in the project was kept physically and emotionally safe at all times during the workshop. The creation of each participant's tartan design began with an acetate-based version, followed by a physical prototype constructed using ribbon, and finally the creation of a digital version using a publically available Internet-based tartan design tool (Figure 1). Each participant was free to determine and shape the tartan design they created during the stages of the design process.

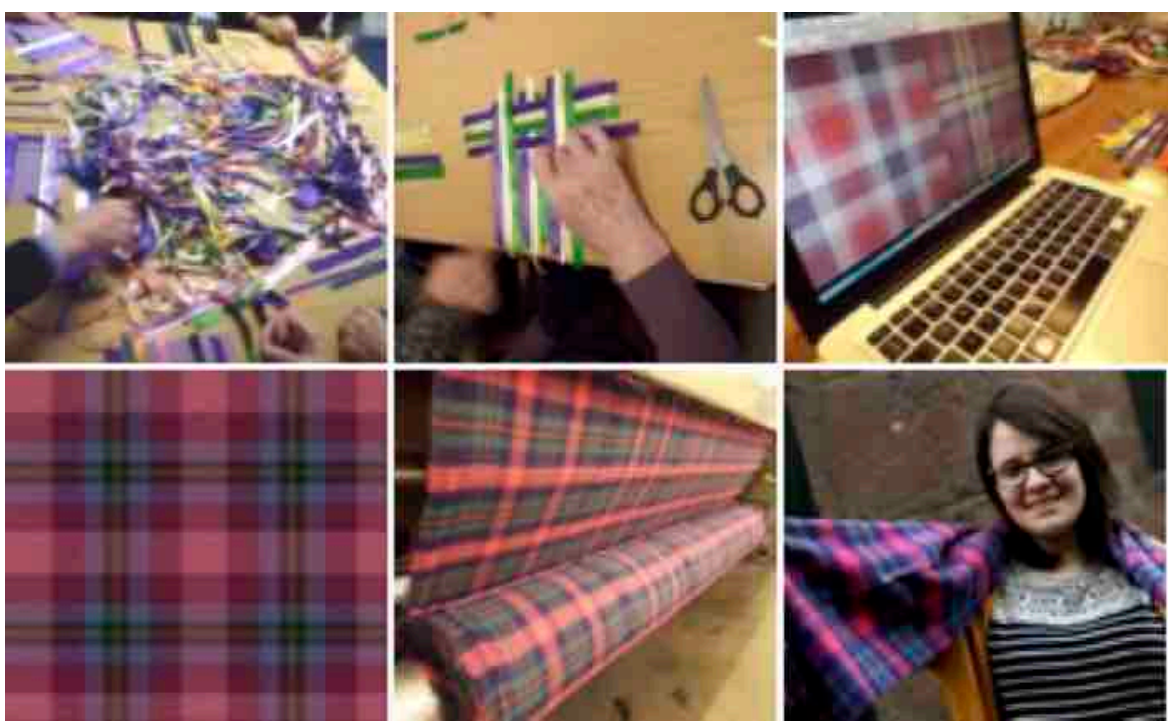

Fig. 1 Tartan design creative process (left to right: acetate, ribbon and digital prototypes)

In the example shown in Figure 1, one can see that the person living with dementia's main colour in their design is purple (Alzheimer Scotland's brand colour), followed by their choice of colours. At this important stage of the co-design 
process, the researchers adopted an empathic (not sympathetic) manner ensuring they were compassionate, un-patronising, tolerant, understanding and respectful. Many of the workshop participants held a significant position before their diagnosis of dementia including an eye surgeon, an architect, and an economist so a respectful attitude was vital to every co-design sessions' success. During the highly iterative stage of co-designing each tartan, the researchers had to consider 'dementia time'. That is, being patient and allowing time and space for each individual and how they might keep track of their time. Many recent approaches to co-design emphasise the need to rethink and redefine the role of the participants (Van Klaveren 2012). For example, one has to be careful not to imply power relations through the terms we use (Holcombe 2010). In this project, the participants are seen as designers in the process and their input is valued as much as the co-design facilitator (authors). Like Manzini and Rizzo (2011), this co-design project views the participants as active collaborative co-designers. The participants are creative; they have a range of experiences, skills, knowledge, and capabilities and they have enhanced the overall nature of the project by taking part. Several authors have proposed nomenclature such as 'vernacular designers' (Reitan 2006), 'silent designers' (Gorb and Dumas 1987) and 'design amateurs' (Leadbeater 2009; Manzini and Rizzo 2011) to describe the co-design participants. It is worth mentioning that embarking on a co-design project not only changes the role of the participants in the design process, it also changes the role of the designer or researcher (Manzini and Rizzo 2011). Most, if not all, of the co-design literature relates (indeed implies) a collaborative and cooperative effort between two or more equally able agents. Many traditional approaches to involve a person in co-design activities, however, create issues as they assume that the participants are cognitively able, can deal with visual and hands-on techniques, and require certain levels of ability. This paper, however, describes codesigning with individuals that are not equal in the sense of their cognitive and communication abilities, which brings new challenges to co-design activities and projects. The remaining sections of the paper report on the significant outcomes of three co-design projects, reflect on the co-designing sessions, and present insights into successful practices when designing together with people living with dementia.

\subsection{Designed With Me}

Designed With $\mathrm{Me}$ is a service design proposition that makes use of the latent creative abilities of an individual's personal knowledge and skills (Kelley and Kelley 2015). Designed With Me adopts a co-design approach where people living with dementia are highly valued and their inputs and collaborations are held in the same esteem as any other participant and collaborator. Designed With Me focusses on the empowerment and inclusion of people living with dementia, along with dementia support workers, carers and the general public, to inform, influence and change local communities. In the process, all participants become co-designers helping to propose possibilities, choose solutions, provide services and "make things happen". Codesign is predominately about 'change' (Brown 2009; Heath and Heath 2011), which relates to making a difference in the world around us. Change scenarios develop the 
collective togetherness in approaching problems and situations. In this scenario, the actions of the designer and the people that they work with are inter-linked by collective responsibility and the desire to make a difference. Work in the Designed With Me project hopes to manifest such change by working with people who have a diagnosis of dementia. The incentive for change and opportunities for co-design do not revolve around resolving the condition of dementia. Rather this work is interested in the power of the individual, the value of their lived experience, and their continuing inclusion in society. Ultimately the purpose of this project is to do stuff with people, to try things out, and to accept occasional failings as long as action has been undertaken and the results evaluated, and used to improve the next approach.

Designed With Me started by exploring the attitudes and opinions of people living with dementia to ascertain what is important for them. This initial stage was undertaken to generate a design brief that would be authored by the people living with dementia themselves. The group, all of whom had a diagnosis of dementia, were invited to respond to everyday questions or statements by filling in answers on the back of a set of designed postcards. The questions invited personal and collective opinion utilising the terms "I" and "We" the purpose of which was to solicit each participant's opinions and to think and talk about their thoughts, hopes, wishes and desires. The initial sessions stimulated a lot of conversation between the authors and a group of people living with dementia, which resulted in free-flowing collective thinking and views peppered with personal insights. The aim of this workshop was to engender a situation where people living with dementia identified areas of potential where design could make a difference. It was also hoped that the design proposal could ultimately have further value and impact in the local community. With this in mind, the postcards used to prompt the group of people living with dementia were openly ambiguous. The open nature of the postcards invited wider thinking, collective discussion and agreement. Gaining the thoughts and wishes of people living with dementia is essential to the goals of this research. This approach, looks to exploit the potential of co-design as a means of raising awareness and developing the voice of people living with dementia. In particular keeping them infused throughout the design process (i.e. formulation of brief, concept generation, development and design delivery). From the first workshop, a number of possible project ideas began to emerge. The considerate and carefully planned approach allowed participants to make explicit their thoughts and considerations. The participants' comments, collected on the postcards, were analysed to identify common themes, thoughts, wants and desires. A number of key themes emerged including:

- Participants wanted more respect and greater communication.

- More appropriate social spaces to meet and mix with others.

- Strong desire to make Sunday special again.

The second co-design workshop focussed on the wants, needs and desires expressed by people living with dementia in the first workshop. That is, participants wished to see greater opportunities to make more time and space for social inclusion, understanding, personal esteem and empowerment, and to be nurtured and 
supported. These key themes were then arranged into a proposition for the second workshop: "Redesigning Sundays to make them special again, where fun can occur that supports respect and communication." One particularly powerful piece of feedback from one of the participants that was offered after the first workshop was: "You made us think more than we are usually asked to do and it is good for us to have to think." Also during the lunch that followed the first workshop, the carers or partners of the individuals involved wanted to know more and to understand what had happened during the session. They were interested in the activity offering their own insights as the discussion continued whilst expressing a clear desire to be involved in future events.
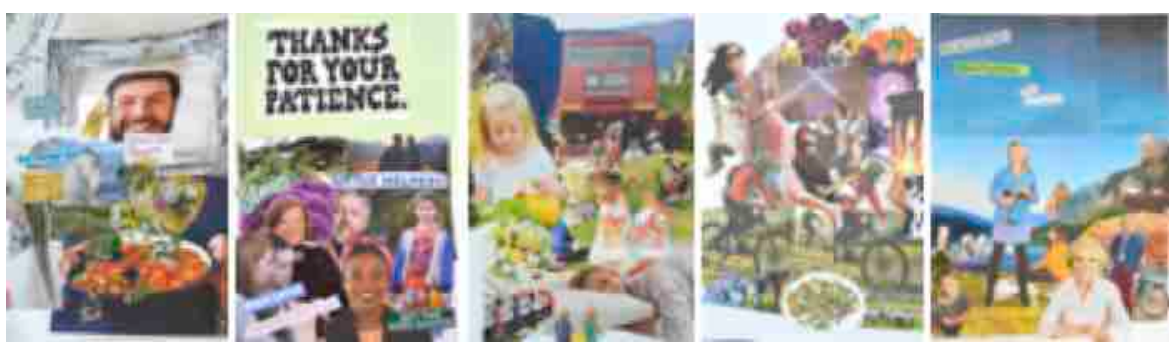

Fig. "Making Sundays special again" initial ideas

The second workshop brought together both carers and people living with dementia to expand upon and propose responses to the brief generated in the first workshop. The larger group (28 participants) assembled in the second workshop allowed for greater involvement, understanding and thinking of all the concerned parties, which supported a sense of togetherness in the discussions and actions. The participants were split into smaller groups of 5 or 6 and each group was invited to make marks, scribble details and stick images down in a collage to encourage discussion and communication of the discussion and emerging project ideas. The ideas proposed in the second workshop, from left to right in Figure 4, are "Family Day", "Water / Boats", "Younger People are a Tonic", "Local Communities and Big Events", and "Encourage Interaction". Currently, the Designed With Me service intervention is considering three concrete proposals with the group of people living with dementia as a response to the brief "Redesigning Sundays to make them special again, where fun can occur that supports respect and communication." The three proposed design interventions under consideration are:

- Our Big Picnic - allows people of all ages and backgrounds to congregate in an organised event and make the entertainment.

- Open Street - will become a local hub for play, talk and local understanding to make the street more like streets from yesteryear.

- D:Caf - where people living with dementia deliver a hospitable place for fun, conversation, innovation, play and companionship. 


\section{$3.375 B C$}

$75 \mathrm{BC}$ is a collaborative design project that celebrates the life and work of the Glaswegian comedian Billy Connolly. The co-design project between the authors and a dementia support group based in Bridgeton in the East End of Glasgow has involved a series of recent visits to the $75 \mathrm{BC}$ murals in Glasgow and to the Amercian artist Tschabalala Self's exhibition at the Tramway Gallery in Glasgow. During this project, five people living with dementia have produced a range of visual representations of Billy Connolly using collage to create a series of textile designs in the style of Tschabalala Self (Figure 5). The artworks created by people living with dementia make use of fabric patterns originally produced in the Bridgeton area of Glasgow (Turkish Red) alongside patterns from the wider creative community. Works by the legendary designer Charles Rennie Macintosh are composed alongside more contemporary designers Timorous Beasties and Laura Spring. Two forms of Billy Connolly have been used here. One image shows Billy Connolly in his iconic Big Banana Boots from early in his career and the other image shows a more recent picture of Billy Connolly at Dressed to Kilt in 2011. All of the the group members composed their patterns and colour schemes in accordance with these two original images. Some followed a clear plan whereas others adopted a much more freeform expression in their representations. The intention of the 75BC co-design project is to use the images created by the five people living with dementia to highlight their inherent creativity and to support the $75 \mathrm{BC}$ celebrations. The members of the dementia support group will decide themselves which of these patterns will become a new Bridgeton textile.

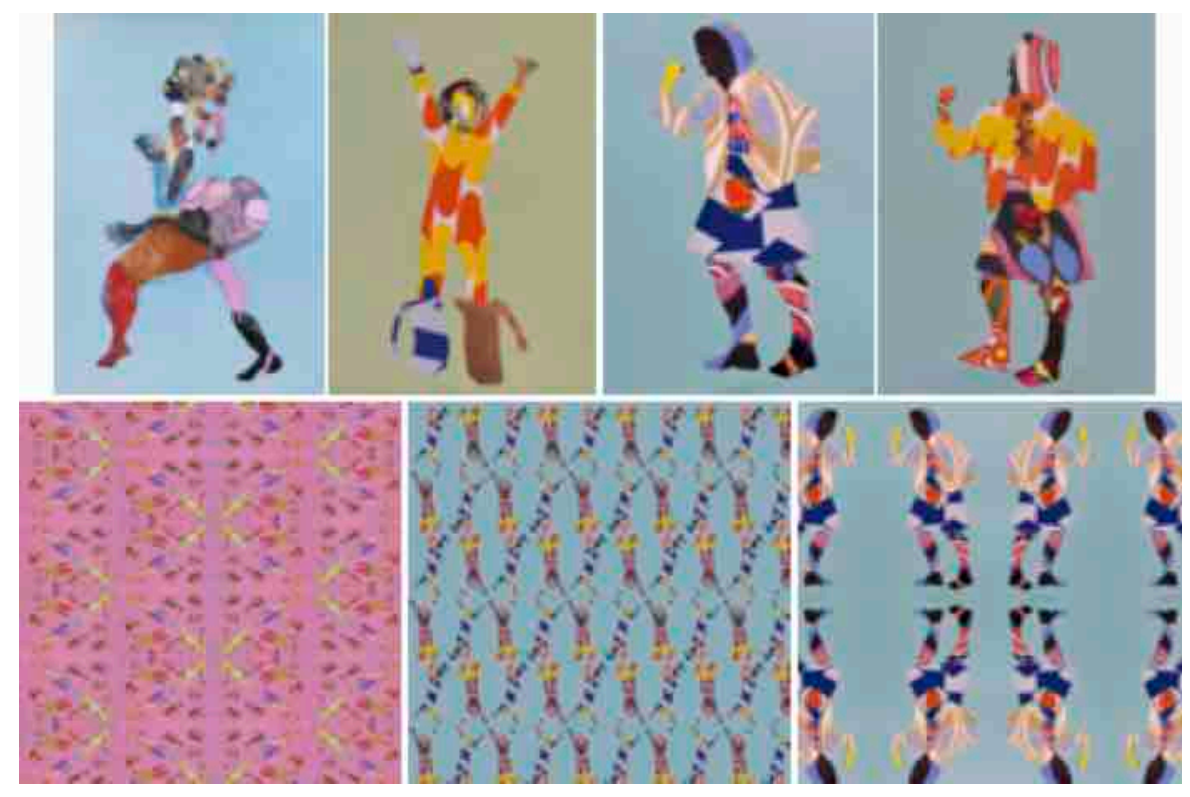

Fig. 5 Tschabalala Self and Billy Connolly collages (top) and New Bridgeton textile fabric design proposals (bottom) 


\section{Conclusions}

Reflecting on these three co-design projects, it is abundantly clear that people living with dementia can offer much to society after diagnosis. Working closely with stakeholders including carers, family members, and collaborating organisations such as Alzheimer Scotland, the authors have received very positive feedback on the codesign projects. Participants have stated that the co-design experiences had been very positive; they have been interested, engaged, and enjoyed the three co-design projects; there had been concentration, focus and discussion during the co-designing activities. Participants felt the three projects had been beneficial. Several of the stakeholders involved considered there to be significant lasting impact for participants in the way the co-design sessions gave people confidence to try new things - some people had been worried about taking part but were very relaxed during the sessions. Impact has also been seen in a number of 'spin-off' projects, discussions, themed activities and outings. Several participants had taken encouragement from the co-design workshops and had since joined a local art group and a number of related spin-off projects have commenced. The three projects presented here show how co-design methods and tools can enable people living with dementia to make a significant contribution to society after diagnosis. Specifically, this work has shown how design thought and action can contribute to changing the perception of dementia and shown that whilst the mood and behaviour of the person may be profoundly affected, their personhood is not. Moreover, the three co-design projects have helped reconnect people recently diagnosed with dementia to build their self-esteem, identity and dignity and keep the person with dementia connected to their local community. The widespread assumption that people living with dementia cannot take part in mainstream activities, and that they have no quality of life or capacity for pleasure and positive involvement has been dismantled by these projects. While the symptoms associated with dementia affect the way a person living with dementia interacts with others, and some activities may be inappropriate as a result, there are many activities such as designing in which they can participate. Moreover, people living with dementia should be encouraged to make decisions or partake in decisions that affect them for as long as possible, to maintain their dignity and self-esteem.

By the year 2030, over $80 \%$ more people aged 65 and over will have some form of dementia (a moderate or severe cognitive impairment) compared to 2010. Design, in general, and design research, in particular, needs to embrace these challenges head on. However, rather than viewing these challenges negatively design has an opportunity to be at the forefront of imagining how we might care and live together better in the future. It is now time, therefore, that design in all its guises grasps this opportunity to envision and realise the future that we will all be proud to share. To envision a future where people living with dementia can make significant contributions to society requires careful consideration and planning. First, researchers should always ask people with dementia how they want to be involved in research, including at what points and in what ways they want to be included. Second, people living with dementia should be involved in setting research priorities. That is, researchers should ask people living with dementia what positive outcomes 
of the research project might look like for them. Third, researchers must ensure that everyone taking part in the research project is physically and emotionally safe at all times. Fourth, researchers must use language that is supportive of people with dementia whilst avoiding language that may offend. Fifth, researchers need to be 'dementia aware'. They should be compassionate, tolerant, understanding, and respectful whilst working with people living with dementia. Lastly, researchers need to consider 'dementia time' in their expectations of research. For instance, finding out the best time to meet and how each individual keeps track of time. Following these guiding principles on how to best conduct co-design projects with people living with dementia will help deliver truly meaningful experiences and outcomes for all involved.

\section{References}

All Party Parliamentary Group on Dementia (2016) Dementia Rarely Travels Alone. APPG on Dementia, London

Alzheimer Scotland (2008) Meeting Our Needs: The Level and Quality of Dementia Support Services in Scotland. Alzheimer Scotland, Edinburgh

Batsch NL, Mittelman MS (2012) World Alzheimer Report 2012: Overcoming the Stigma of Dementia. Alzheimer's Disease International, London

Brown T (2009) Change by Design: How Design Thinking Transforms Organisations and Inspires Innovation. HarperCollins, New York

Bury M (1982) Chronic Illness as Biographical Disruption. Sociology of Health \& Illness 4 2: $167-182$

Christensen C, Overdorf M (2000) Meeting the Challenge of Disruptive Change. Harvard Business Review 78 2: 66-76

Donetto S, Pierri P, Tsianakas V, Robert G (2015) Experience-based Co-design and Healthcare Improvement: Realizing Participatory Design in the Public Sector. The Design Journal 18 2: 227-248

Gorb P, Dumas A (1987) Silent Design. Design Studies 8 3: 150-156

Heath C, Heath D (2011) Switch: How to Change Things When Change is Hard. Random House, London

Hendriks N, Slegers K, Duysburgh P (2015) Codesign with People Living with Cognitive or Sensory Impairments: A Case for Method Stories and Uniqueness. CoDesign 11 1: 70-82

Holcombe S (2010) The Arrogance of Ethnography: Managing Anthropological Research Knowledge. Australian Aboriginal Studies 2: 22-32

Kanstrup AM (2016) Living in the Lab: An Analysis of the Work in Eight Living Laboratories Set Up in Care Homes for Technology Innovation. CoDesign 13 1: 49-64

Katsuno T (2005) Dementia from the Inside: How People with Early-stage Dementia Evaluate their Quality of Life. Ageing and Society 25: 197-214

Kelley T, Kelley D (2015) Creative Confidence: Unleashing the Creative Potential Within Us All. William Collins, London

Kinnaird L (2012) Delivering Integrated Dementia Care: The 8 Pillars Model of Community Support, September. Alzheimer Scotland, Edinburgh

Kitwood T (1990) The Dialectics of Dementia: With Particular Reference to Alzheimer's Disease. Ageing \& Society 10: 177-196

Lam B, Dearden A, William-Powlett K, Brodie E (2012) Exploring Co-design in the Voluntary Sector. In Proceedings of VSSN/NCVO Annual Conference, University of Birmingham, Birmingham, AL 
Leadbeater C (2009) We Think. Profile Books, London

Manzini E, Rizzo F (2011) Small Projects/Large Changes: Participatory Design as an Open Participated Process. CoDesign 7 3-4: 199-215

Matthews E (2006) Dementia and the Identity of the Person. In Dementia: Mind, Meaning and the Person, Hughes JC, Louw SJ, Sabat SR (eds.), Oxford University Press, Oxford, 163-177

Office for National Statistics (2017) Overview of the UK population: July 2017. ONS, London

Prince M, Prina M, Guerchet M (2013) World Alzheimer Report 2013. Journey of Caring: An Analysis of Long-term Care for Dementia. Alzheimer's Disease International, London

Reitan JB (2006) Inuit Vernacular Design as a Community of Practice for Learning. CoDesign 2 02: 71-80

Rodgers PA, Tennant A (2014) Disrupting Health and Social Care by Design. In Proceedings of the 9th International Conference on Design \& Emotion, Bogota, Colombia

Sanders EB-N, Stappers PJ (2014) Probes, Toolkits and Prototypes: Three Approaches to Making in Codesigning. CoDesign 10 1: 5-14

Scharmer CO (2011) Leading from the Emerging Future. In Minds for Change - Future of Global Development Ceremony to mark the 50th Anniversary of the BMZ Federal Ministry for Economic Cooperation and Development, Berlin

Van Klaveren R (2012) Artistic Participatory Practices as a Vehicle for Togetherness. In Proceedings of the CUMULUS Conference, Helsinki, 1-11

Wildevuur SE, van Dijk D (2011) Scottie: Design for Social Connectedness in Healthcare. CoDesign $72: 131-138$

Wilson S, Roper A, Marshall J, Galliers J, Devane N, Booth T, Woolf C (2015) Codesign for People with Aphasia Through Tangible Design Languages. CoDesign 11 1: 21-34 